\title{
Les pratiques de « résistance » dans les malls de Rabat-Salé: accessibilité et usage social d'un espace sélectif
}

"Resistance" Practices in the Shopping Malls of Rabat-Sale: Accessibility and the Social Use of a Selective Space

Tarik Harroud

\section{(2) OpenEdition Journals}

\section{Édition électronique}

URL : http://journals.openedition.org/conflits/19153

DOI : $10.4000 /$ conflits. 19153

ISSN : $1777-5345$

\section{Éditeur :}

CCLS - Centre d'études sur les conflits lilberté et sécurité, L'Harmattan

\section{Édition imprimée}

Date de publication : 19 mai 2016

Pagination : $57-79$

ISBN : 978-2-343-09459-5

ISSN : 1157-996X

\section{Référence électronique}

Tarik Harroud, «Les pratiques de « résistance » dans les malls de Rabat-Salé: accessibilité et usage social d'un espace sélectif », Cultures \& Conflits [En ligne], 101 | printemps 2016, mis en ligne le 19 mai 2017, consulté le 30 mars 2021. URL : http://journals.openedition.org/conflits/19153 ; DOI : https:// doi.org/10.4000/conflits. 19153 


\title{
Les pratiques de "résistance » dans les malls de Rabat-Salé : accessibilité et usage social d'un espace sélectif
}

\section{Tarik HARROUD}

Tarik Harroud est architecte-géographe, enseignant-chercheur à l'Institut national d'aménagement et d'urbanisme de Rabat au Maroc. Il a soutenu en 2013 une thèse de doctorat sur les restructurations spatiales et sociales des centres commerciaux dans la capitale marocaine et a publié notamment: "De la Kissaria au Méga Mall: de nouvelles formes de sociabilité juvéniles à Rabat " in Dupret et al (dir.), Le Maroc au présent, publication de la fondation Al Saoud et CJB, 2016 ; "L'arrivée des centres commerciaux dans les marges urbaines de Rabat: Des lieux inédits de sociabilité et de déambulation urbaine ", L’Année du Maghreb, 12, 2015 ; Processus de production des villes nouvelles au Maroc, Publications de l'INAU, 2010 ; "Les nouveaux espaces marchands dans la périphérie de Rabat: Usages, représentations et nouvelles urbanités ", Les Cahiers d'EMAM, 18, 2009.

\begin{abstract}
À l'instar de la majorité des pays du monde arabe, le Maroc connaît, depuis

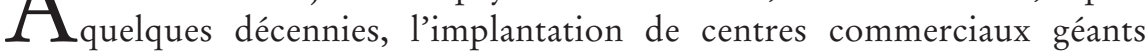
conçus sur le modèle des malls ${ }^{1}$ américains. Ces équipements se sont rapidement multipliés au point de marquer le paysage commercial et urbain de la plupart des grandes villes du pays ${ }^{2}$. C'est particulièrement dans les périphéries urbaines, espaces de plus en plus privilégiés pour leurs disponibilités fon-
\end{abstract}

1. L'expression mall est un terme américain (issu de l'appellation courante de shopping mall) désignant un centre commercial articulé autour d'un axe de distribution couvert, fermé et climatisé. Cette expression est souvent confondue au Maroc tant par les médias que dans la nomenclature officielle du ministère du Commerce et de l'Industrie avec les autres types de commerce moderne (grandes surfaces alimentaires, grandes surfaces spécialisées d'ameublement). Dans cette contribution, le terme mall renvoie à des centres commerciaux géants ayant fait leur apparition au Maroc au cours des années 2000 et dont la vocation reste principalement récréative et ludique avec un rôle de locomotive confié à des espaces de shopping et de loisirs.

2. Harroud T., Émergence de nouvelles centralités commerciales à Rabat : sociabilités et restructurations spatiales, thèse de doctorat en géographie, Université Mohammed V, Rabat, 2013. 
cières et leur accessibilité, que ces dynamiques sont saisissantes à observer et à étudier. Impulsés par de grandes firmes nationales et internationales dans le cadre d'importants investissements financiers, ces équipements, souvent insérés dans des réseaux à l'échelle mondiale ${ }^{3}$, sont le signe de l'introduction du Maroc dans l'économie néolibérale, amorcée depuis la fin des années 1980 suite aux effets de la politique d'ajustement structurel et accélérée avec l'arrivée du nouveau roi ayant placé, juste après son intronisation, l'investissement comme priorité nationale. Profitant tout autant d'une série d'importantes incitations financières et fiscales que d'une conjoncture économique et sociale favorable (amélioration des conditions de vie de la population, émergence d'une classe moyenne dans les grandes villes, augmentation sensible des taux de motorisation, etc.), ces grandes chaînes étrangères, en association avec des firmes nationales, ont fait du Maroc un territoire privilégié pour étoffer leur réseau international et développer des malls aux formats et aux concepts commerciaux hétérogènes ${ }^{4}$. Avec une offre commerciale très dense qui s'étend sur de grandes superficies, ces malls mobilisent, pour leur réalisation, des fonds financiers considérables qui justifient le recours par leurs promoteurs à des logiques de rentabilité et de maximisation du profit ${ }^{5}$. Ainsi, ces derniers élaborent en amont de leur création, des études de faisabilité économique et financière ainsi que des études de marché et d'identification des zones de chalandise ${ }^{6}$. Ces logiques impliquent souvent, comme il a été révélé dans plusieurs contextes étrangers ${ }^{7}$, le recours à des dispositifs implicites de ciblage et de sélectivité d'une clientèle « solvable » répondant aux critères commerciaux

3. Vignal L., «Beyrouth, de la boutique au shopping mall: dynamiques métropolitaines et nouvelle géo-économie au Moyen-Orient ", in Peraldi M., Mermier F. (dir), Mondes et places $d u$ marché en Méditerranée : Formes sociales et spatiales de l'échange, Paris, Karthala, 2011.

4. Dans un souci de clarification de la terminologie utilisée pour l'appellation des espaces marchands abordés dans cette contribution (centre commercial et mall), nous faisons une distinction entre le centre commercial renvoyant aux équipements marchands à vocation alimentaire et comportant, notamment, une grande surface alimentaire de type hypermarché ou supermarché et le mall renvoyant au centre commercial à vocation récréative et faisant des lieux de loisirs et des marques internationales ses espaces locomotifs. Dans le cas de Rabat, il existe deux malls qui sont déjà réalisés (Méga Mall et Marjane Ryad, centre commercial reconverti en 2010 en mall). Depuis la fin de la décennie 2000, on assiste à la multiplication des projets de création de malls géants qui sont en cours de réalisation (Rabat Center, Rabat Bouregreg, Mahaj Ryad, etc.).

5. Sorkin M., Shopping Mall, from Variations on a Theme Park, The New American City and the End of Public Space, New York, Hill and Wang, 1992.

6. Il s'agit des zones d'attraction définies par les promoteurs commerciaux en fonction d'un ensemble de critères sociaux, économiques et spatiaux et d'où provient la majorité de la clientèle ciblée.

7. Citons au sujet de l'accessibilité sociale des centres commerciaux et des dispositifs sélectifs de leur accès, les travaux conduits notamment par Guénola Capron à Buenos Aires en Argentine (Capron G., "Les centres commerciaux à Buenos Aires. Les nouveaux espaces publics de la ville de la fin du XXe siècle ", Les Annales de La Recherche Urbaine, n78, 1998, pp. 59-65), Bruno Sabatier au Mexique (Sabatier B., «Les complexes commerciaux récréatifs en France et au Mexique : une (ré)-intégration du temps de loisir au temps d'achat», in Bondue J.-P. (dir.), Temps des courses, course des temps, CNFG, Université des Sciences et Technologies de Lille, 2004) et Myriam Houssay-Holzschuch en Afrique du Sud (Houssay-Holzschuch M., Teppo A., " A mall for all? Race and public space in post-apartheid Cape Town », Cultural geographies, $\mathrm{n}^{\circ} 16,2009$, pp. 351-379. 
définis en amont de leur création. Par ailleurs, elles interpellent la question de l'accessibilité sociale de ces équipements pour les différentes catégories de population dans un contexte local urbain marqué par une forte fragmentation sociale et spatiale ${ }^{8}$. Cette question de l'accessibilité sociale des centres commerciaux, qui ne désigne pas seulement « les potentialités de mise en relation entre des lieux (ce qui renverrait à l'accessibilité physique et géométrique, liée aux réseaux de transport), mais aussi le degré de porosité et d'hospitalité d'un lieu, d'accueil d'usages et de publics pluriels 9 » reste posée d'autant plus que ces derniers sont souvent présentés par leurs promoteurs comme des lieux ouverts et gratuits prolongeant les lieux marchands publics des villes marocaines. À partir du contexte spécifique de l'agglomération de Rabat-Salé ayant connu, au cours des deux dernières décennies, un développement rapide et remarquable de ces équipements marchands, l'article aura pour but de mettre en lumière les représentations symboliques et les rapports sociaux qu'entretiennent les groupes sociaux à faible pouvoir d'achat, issus des marges urbaines, avec ces vitrines de l'Occident ${ }^{10}$, souvent associées par les jeunes Marocains aux référents de «kharij » (l'étranger) et «barra " (extérieur) très appréciés par la plupart d'entre eux ${ }^{11}$. La présente contribution s'inscrit aussi dans le sillage de nombreux travaux de recherche abordant, dans le contexte actuel de la mondialisation de l'économie et de la culture ${ }^{12}$, la diffusion dans les pays en voie de développement ou " périphériques » de modèles et d'espaces transnationaux - allant de pair avec l'arrivée de nouveaux acteurs internationaux dans la fabrication et l'aménagement des villes des Suds - ainsi que les modalités de leur insertion locale, spatiale et socioculturelle.

Elle cherche ainsi à étudier, dans le cadre d'une anthropologie du néolibéralisme ${ }^{13}$, l'incarnation microphysique du néolibéralisme ${ }^{14}$ dans l'une de ses

8. Voir les travaux de Navez-Bouchanine et Abu-Lughod sur la fragmentation sociale et spatiale dans les villes marocaines : Navez-Bouchanine F., Fragmentation spatiale et urbanité dans les villes maghrébines, rapport de recherche (1995-1996) établi pour le Programme interdisciplinaire de recherche sur la ville, Laboratoire de recherche URBAMA, Tours, 1997 et Lughod J., Rabat: Urban Apartheid in Morocco, Princeton, Princeton University Press, 1980.

9. Capron G., "L'accès aux espaces publics modernes dans les villes latino-américaines : Apparences physiques et réalités socio-spatiales ", in Actes de la Conference "Rights to the City", International Geographical Union, Rome, May 29-June 1, 2002, Home of Geography Publication Series, vol. III, p. 6.

10. Bennani-Chraïbi M., "Jeunes Égyptiens et jeunes Marocains face à l'Occident ", Égypte/Monde arabe, Première série, $\mathrm{n}^{\circ} 30-31,1997$, mis en ligne le 8 juillet 2008, consulté le 14 juin 2012.

11. Harroud T., "L'arrivée des centres commerciaux dans les marges urbaines de Rabat: Des lieux inédits de sociabilité et de déambulation », in Belguidoum S, Cattedra R., Iraki A. (dir.), "Villes et urbanités au Maghreb ", L’Année du Maghreb, n¹2, 2015, pp. 75-89.

12. Warnier J-P., Mondialisation de la culture, Paris, La Découverte, 1999.

13. Elle s'inscrit également dans le cadre d'une anthropologie des mondes contemporains dont parle Marc Augé qui appelle, face aux mutations de ce monde contemporain, à revisiter l'anthropologie et à étendre ses sujets et ses terrains de recherche pour englober de nouveaux domaines réservés jadis à d'autres disciplines (Augé $\mathrm{M}$, Pour une anthropologie des mondes contemporains, Paris, Aubier, 1994).

14. Nous contribuons ici aux différentes réflexions faites à ce sujet par un ensemble de chercheurs ayant abordé, à partir de plusieurs entrées analytiques (centres commerciaux, urbanisme néo- 
manifestations spatiales les plus emblématiques à l'échelle mondiale, à savoir les centres commerciaux géants. Nous nous intéressons plus précisément aux réactions sociales et symboliques exprimées par le bas, c'est-à-dire par les populations ordinaires issues des marges urbaines dans un pays en bordure de la mondialisation, par rapport à ces morceaux de territoire globalisé.

Ces équipements marchands représentent en effet des espaces pertinents à analyser dans la mesure où ils sont révélateurs d'une nouvelle modernité ${ }^{15}$, voire d'une surmodernité 16 telle qu'elle est définie par Marc Augé qui n’hésite pas à les qualifier d'ailleurs de "non-lieux 17 ", c'est-à-dire d'espaces non anthropologiques, interchangeables, sans âme et dépourvus de cette dimension identitaire, relationnelle et historique propre aux « lieux » urbains. Cela s'oppose aux significations du concept de lieu, tel qu'il est appréhendé par bon nombre de géographes qui l'associent au principe de coprésence et d'interaction sociale. Il représente, à la suite de Michel Lussault ${ }^{18}$, une petite unité spatiale complexe, circonscrite et délimitée favorisant des coprésences et des interactions entre les éléments qui les composent.

Bien qu'ils représentent un objet de recherche relativement récent, les centres commerciaux dans le monde arabe ont suscité, par ailleurs, l'intérêt de plusieurs chercheurs d'horizons disciplinaires divers ${ }^{19}$ ayant exploré diffé-

libéral, grands projets urbains, espace public, etc.), les effets sociaux et spatiaux du néolibéralisme dans les villes du tiers monde. Nous citons particulièrement les travaux de Mathieu Hilgers sur l'urbanisme néolibéral et ses effets territoriaux en Afrique (Hilgers M., « À qui appartient la ville ? Urbanisme néolibéral et propriété dans trois petits centres urbains du Ghana et du Burkina Faso ", Politique africaine, n¹32, 2013, pp. 95-113), de Mike Davis et Yasser Elsheshtawy dans la péninsule arabique particulièrement à Dubai (Davis M., Monk D., Paradis infernaux. Les villes hallucinées du néo-capitalisme, Paris, Les prairies ordinaires, 2007 ; Elsheshtawy Y., "Redrawing Boundaries: Dubai, The emergence of a Global city », in Elsheshtawy Y. (ed.), Planning the Middle East City: An Urban Kaleidoscope in a Globalizing World, London, 2004, pp. 167-199), de Guénola Capron et Jérôme Monnet en Amérique latine (Capron G., "Les centres commerciaux à Buenos Aires. Les nouveaux espaces publics de la ville de la fin du XXe siècle ", op.cit.; Monnet J. (dir.), L'urbanisme dans les Amériques. Modèles de ville et modèles de société, Paris, Karthala, 2000) et enfin les travaux de Bénédicte Florin et Nora Semmoud dans la zone méditerranéenne (Semmoud N. et al., Marges urbaines et néolibéralisme en Méditerranée, Tours, Presses universitaires François Rabelais, 2014).

15. Freitas R-F., Centres commerciaux : îles urbaines de la postmodernité, Paris, L'Harmattan, 1996.

16. Il s'agit d'un concept forgé par Marc Augé pour signifier un dépassement de la modernité. Il choisit le suffixe « sur » pour mettre l'accent sur l'idée d'excès liée à une accélération excessive du temps et une profusion d'événements, excès de l'espace qui se caractérise par une prolifération de non-lieux et enfin excès d'individus marqué par une trop grande individualisation des références.

17. Selon lui les «non-lieux » se définissent «par opposition à la notion sociologique de lieu, associée par Mauss et toute une tradition ethnologique à celle de culture localisée dans le temps et l'espace » (Augé M., Non-Lieux, Introduction à une anthropologie de la sur modernité, Paris, Seuil, 1992, p. 48).

18. Lussault M., L'bomme spatial : La construction sociale de l'espace humain, Paris, Le Seuil, 2007.

19. Il n'est pas question ici de citer l'ensemble de ces travaux d'autant plus que l'intérêt porté à cet objet s'accroît au sein du monde arabe. Nous faisons particulièrement référence à l'ou- 
rentes dynamiques sociales, spatiales et culturelles de l'insertion de ces équipements dans le paysage urbain des grandes villes arabes.

Tout en nous inscrivant dans le sillage de ces travaux, nous comptons toutefois centrer notre propos, sur une dimension peu investie par ces essais et portant sur la question de la « résistance 20 » au sein de ces structures marchandes. Nous l'associons aux différentes pratiques d'appropriations et de détournements ${ }^{21}$ des logiques normatives et marchandes des centres commerciaux 22 .

Au-delà des pratiques visibles de résistance observées dans ces lieux, il s'agira, dans cette contribution, de mettre en exergue, également, les arts de faire ${ }^{23}$ et les arts de croire (en tant que pratiques de résistance muettes et discrètes) déployés par les populations à faibles revenus issues des quartiers modestes du Grand Rabat et, plus particulièrement, par des catégories spécifiques comme les jeunes et les femmes ${ }^{24}$, pour investir et s'approprier socialement et symboliquement ces espaces marchands et sélectifs.

Sur la base d'une série d'entretiens semi-directifs 25 et de séances d'observation conduites sur un échantillon de centres commerciaux de l'agglomération, l'article met en exergue trois formes de résistance renvoyant à trois principales représentations et images qui leur sont associées :

vrage collectif dirigé par Michel Peraldi et Franck Mermier, fruit d'un programme de recherche 2009-2010 portant sur les espaces et rapports marchands en Méditerranée (Peraldi M., Mermier F., Mondes et places du marché en Méditerranée, op. cit.) qui comporte un ensemble de travaux empiriques sur l'implantation des malls dans quelques villes arabes (Le Caire, Amman, Beyrouth, Dubaï, Tunis, Ryad, Sanaa). Une bonne partie de ces travaux met l'accent sur les nouvelles formes de sociabilité des femmes et leur accès à l'espace public.

20. Hollander J., Einwohner R., «Conceptualizing Resistance », Sociological Forum, vol. 19, n4, 2004, pp. 533-554.

21. Nous appréhendons cette dimension comme une forme d'appropriation sociale assimilée à une résistance et exprimée par des sujets à l'encontre de normes et règles de conduite qui leur sont imposées et qui s'en saisissent pour l'asservir à leurs propres fins.

22. Harroud T., «Les nouveaux espaces marchands dans la périphérie de Rabat », Les Cabiers d'EMAM, n`18, 2009, pp. 69-88.

23. De Certeau M., L'invention du quotidien. 1: Arts de faire, Paris, Gallimard, 1980.

24. On renvoie ici aux travaux conduits sur l'accès des femmes arabes aux espaces publics et aux équipements marchands. On cite, entre autres, les travaux conduits par Amélie Le Renard en Arabie Saoudite, Mona Abaza en Égypte, Davis Hannah au Maroc et Laure Assaf au Liban et aux Émirats Arabes Unis : Le Renard A., Femmes et espaces publics en Arabie Saondite, Paris, Dalloz, 2011 ; Abaza M., "Egyptianizing the american dream, Nasr city's shopping malls, public order, and the privatized military», in Singerman D., Amar P. (eds), Cairo Hegemonic: State, Justice, and Urban Social Control in the New Middle East, Cairo, American University of Cairo Press, 2009, pp. 193-221; Hannah D-T., «"Là où vont les femmes" : notes sur les femmes, les cafés, et les Fast Food au Maroc ", Cahiers de recherche, n 4, 1995, pp. 11-18; Assaf L., "Espaces vécus et imaginés des rencontres amoureuses aux Émirats arabes unis », EchoGéo, n²5, 2013, en ligne, consulté le 15 janvier 2016.

25. Entre 2010 et 2012, des entretiens avec les promoteurs et un échantillon de visiteurs ont été conduits dans les principaux malls et centres commerciaux de Rabat-Salé ; s'y sont ajoutées des séances d'observation denses sur les modalités de leur fréquentation et utilisation sociale. 
- Résistance «pour » le mall perçu comme espace « ressource » pour lequel des profils sociaux à faible pouvoir d'achat déploient un ensemble de pratiques sociales afin de pouvoir s'y rendre et y déambuler aisément. Le loisir ici, peut être compris comme forme de résistance.

- Résistance «par » le mall perçu comme espace "refuge » dans lequel les catégories juvéniles et féminines peuvent surmonter et s'affranchir du contrôle social imposé par leurs milieux d'origine.

- Résistance «contre » le mall perçu par d'autres profils sociaux en tant qu'espace « intrus » qui met en danger les valeurs sociales, culturelles et identitaires locales.

\section{Une nouvelle géographie commerciale se dessine dans l'agglomération Rabat-Salé}

À l'instar des grandes villes du pays, le Grand Rabat a vu fleurir au cours des deux dernières décennies, un nombre considérable de malls et de centres commerciaux qui contribuent à diversifier son paysage commercial.

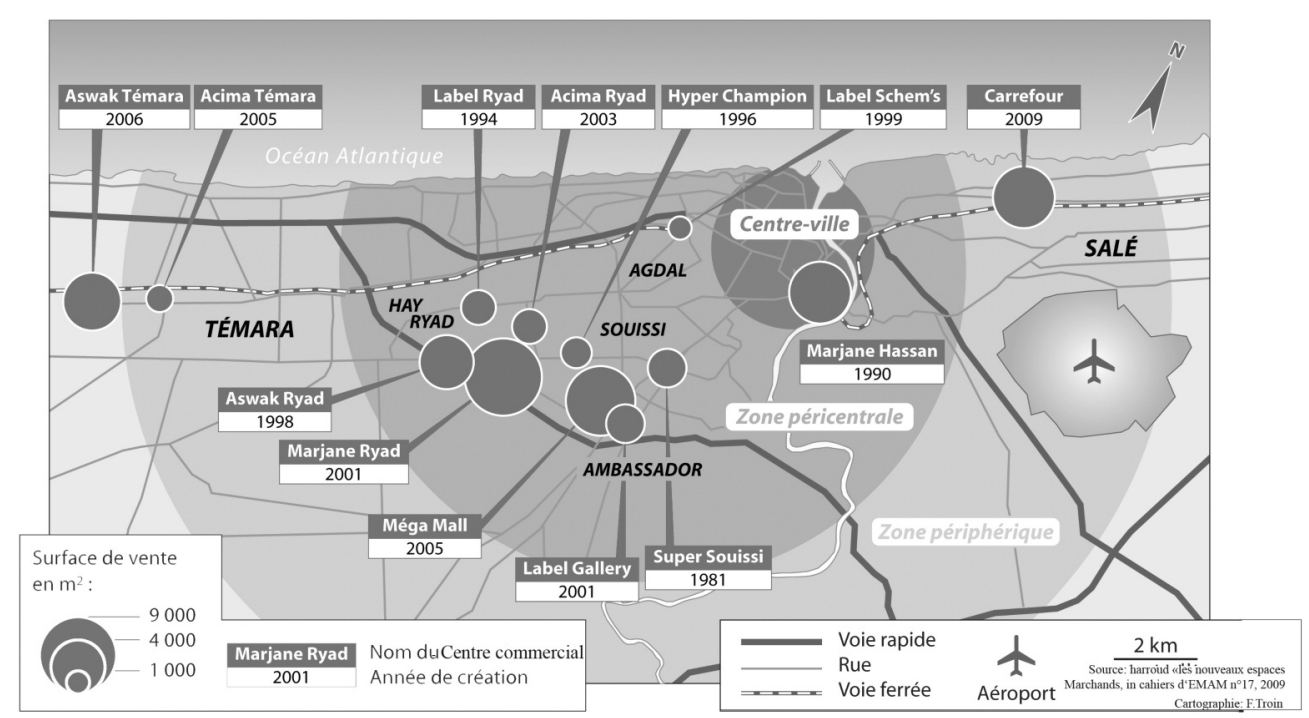

Répartition des centres commerciaux à Rabat-Salé avec les dates de leur création (source : élaboration personnelle 2013)

Ces espaces marchands ont trouvé dans la capitale les conditions favorables à leur multiplication. L'essor d'une économie tertiaire, conjuguée à la montée fulgurante de la classe moyenne, ainsi que l'amélioration des infrastructures routières et de transport (création de voies express et de voies de contournement) sont parmi les facteurs favorisant leur développement. Aujourd'hui, la capitale se positionne en seconde place à l'échelle nationale en 
ce qui concerne la concentration d'établissements marchands, puisqu'on y dénombre une vingtaine d'équipements commerciaux.

Après avoir initialement privilégié des localisations péricentrales à l'interface des voies structurantes de capitale et à proximité des quartiers huppés (Agdal, Soussi et Hay Ryad), ces structures essaiment progressivement vers les différentes couronnes au fil du développement de l'agglomération et de la création de nouvelles rocades pour s'implanter, ensuite, dans les couronnes périphériques de Salé et de Témara sans pour autant que cette implantation soit suivie par une desserte en transports en commun. On peut proposer une typologie des principaux centres commerciaux de l'agglomération, ventilée en trois types d'équipements : les malls géants à vocation récréative et ludique situés dans les quartiers huppés de Rabat et ciblant une clientèle aisée (Méga Mall, Marjane Ryad, Kitéa Géant Ryad) ; les grands centres commerciaux alimentaires à forte attractivité situés dans les zones péricentrales de l'agglomération et ciblant une clientèle de la classe moyenne (Aswak Assalam, Marjane Bouregreg, Acima Ryad, Label'Vie Galery à Souissi, etc.) ; les centres commerciaux alimentaires situés dans les communes périphériques de Rabat et ciblant un public hétérogène (Carrefour Salé, Marjane Tabriquet à Salé, Asswak Assalam Témara, etc.).

En attirant de façon progressive de nombreuses enseignes et marques commerciales localisées auparavant dans les quartiers centraux, ces nouveaux équipements marchands sont en train de dessiner une nouvelle géographie commerciale à l'échelle de l'agglomération 26.

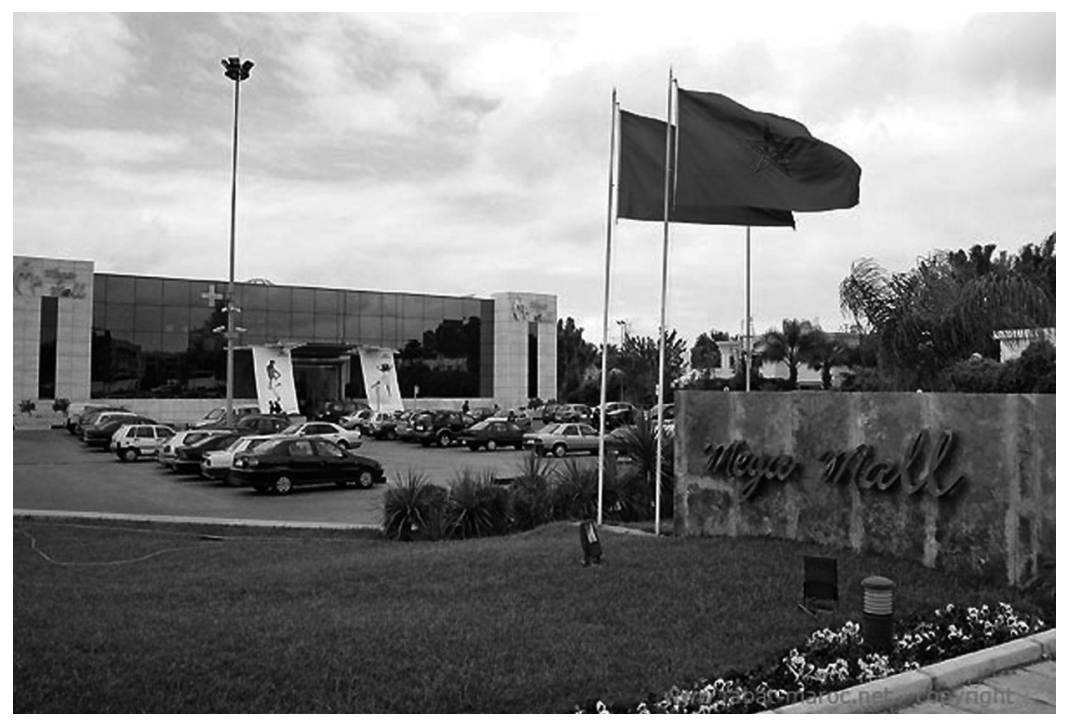

Méga mall situé dans le quartier huppé de Rabat

26. Harroud T., «Émergence de nouvelles centralités commerciales », op. cit. 
Réalisés sur de grandes superficies avec des offres commerciales très consistantes, ces centres marchands mobilisent pour leur réalisation des montants d'investissement considérables. Cela explique le souci majeur de rentabilité affiché par leurs promoteurs qui mettent en place tout un ensemble de dispositifs commerciaux, spatiaux et sécuritaires permettant de maximiser les profits et de garantir une pérennité maximale à leurs magasins. Ces dispositifs renseignent sur les modes et les normes de fonctionnement de ces équipements, mais aussi sur leur niveau d'ouverture ou d'accessibilité auprès des différentes catégories sociales de l'agglomération Rabat-Salé.

L'un des premiers aspects par lequel peut être appréciée l'accessibilité de ces espaces concerne la nature et le standing de leur offre commerciale. Si l'on peut caractériser globalement cette dernière par sa diversité dans la mesure où le commerce banal se combine au commerce de luxe, elle reste majoritairement dominée par l'offre de produits « haut de gamme » (commerces rares, franchises et marques internationales, restaurants et cafés «branchés », etc.) qui témoignent de leur niveau d'accessibilité. Cette offre " haut de gamme ", associée à une mise en scène spatiale et architecturale, inspire le luxe : choix de matériaux luxueux, installation d'escalators et ascenseurs transparents, mise en scène par les jeux d'éclairage, etc. ${ }^{27}$ Conçue le plus souvent par des architectes renommés, l'ambiance crée un univers raffiné et d'opulence recherché par les promoteurs ${ }^{28}$. En raison de la prédominance de ces enseignes de prestige et des offres commerciales onéreuses qu'elles exposent, il est évident que la clientèle visée par ces malls est constituée de catégories sociales à fort pouvoir d'achat pouvant s'offrir les produits de luxe qui y sont en vente.

Dans cette stratégie de ciblage, les catégories à faible pouvoir d'achat ne sont pas systématiquement exclues. Toutefois, leurs poids doit rester minoritaire dans le but de préserver l'image de prestige prônée par les promoteurs commerciaux ${ }^{29}$. Cela renvoie au dosage subtil de l'ouverture/fermeture de ces espaces qui, tout en permettant l'accès élargi à la clientèle locale, met en place tout un ensemble de dispositifs normatifs et sécuritaires limitant un accès qui serait trop généralisé et jugé « nuisible » à leur image de standing. "L’image du mall est notre capital, c'est la garantie de sa pérennité et le moyen de fidéliser notre clientèle. Nous ouvrons par tous les moyens à la préserver 30 » confirme le directeur de Méga Mall. Le second élément par lequel peut être appréciée l'accessibilité sociale et physique de ces lieux se rapporte au choix

27. À l'instar des malls géants de la péninsule arabique, il s'agit souvent d'architectures monumentales qui véhiculent l'idée à la fois de gigantisme mais aussi d'abondance et d'opulence. À ces mises en scène architecturales, s'ajoutent des dispositifs normatifs et sécuritaires (vigiles et caméras de surveillance) qui véhiculent l'idée d'un espace sécurisé et « sélect ».

28. Voir sur ce sujet de l'ambiance des malls l'article de Margaret Crawford : Crawford M., «The World in a Shopping Mall », in Sorkin M. (ed.), Variations on a Theme Park, op. cit., 1992, pp. 3-30.

29. Harroud T., «Émergences de nouvelles centralités commerciales », op. cit.

30. D’après l'entretien réalisé avec Harim, le directeur de Méga Mall, 2010. 
du site de leur implantation : il se fait suivant des critères liés à la facilité d'accès et à la visibilité. De telles considérations donnent l'avantage soit - dans une logique de proximité spatiale - à des zones péricentrales situées à proximité des quartiers aisés du Grand Rabat, soit à des zones périphériques - dans une logique de proximité temporelle - très bien connectées aux voies rapides de l'agglomération. Dans cette logique de localisation, il est évident que l'accès par la voiture reste le moyen d'accès privilégié et dominant par rapport aux autres moyens de transport. L'inscription spatiale des principaux centres commerciaux de l'agglomération par rapport au réseau de transport en bus le montre bien car seules quelques lignes de bus à faible fréquence desservent quelques-uns de ces malls. Ceci constitue un autre obstacle à l'accès des catégories sociales modestes non motorisées. Interrogé à ce propos, le directeur de Marjane Ryad n'hésite pas à pointer la nécessité de venir en voiture pour faire de gros achats : "C'est évident que, pour venir ici à Marjane, il faut être motorisé, tous les centres commerciaux du monde fonctionnent de cette manière et puis on ne peut pas apporter les approvisionnements de grande quantité en bus, c'est impossible 31 ».

Par ailleurs, si les dispositifs de surveillance dans ces malls sont souvent présentés par les promoteurs comme des arrangements permettant d'assurer la sécurité et le bien être des clients et d'éviter toute forme de nuisances ou de comportements déviants ${ }^{32}$, ils cachent toutefois des logiques implicites de «filtrage » à l'encontre des personnes indésirables dont le profil ne s’inscrit pas dans la clientèle ciblée. Les gestionnaires de ces espaces refusent généralement de reconnaître directement la pratique du filtrage, qui doit rester la plus discrète possible pour entretenir l'image d'accessibilité universelle de leurs espaces. Ils évoquent souvent les questions de l'ordre et le confort des consommateurs qui dissimulent en tant que telles d'autres considérations. Certes, il est extrêmement rare d'identifier dans ces équipements des situations de refus d'accès ou d'expulsion visibles des personnes qui les fréquentent 33 (à cause de leur situation socio-économique ou de leur apparence phy-

31. D’après l'entretien réalisé avec Omar, ancien directeur du Marjane Ryad.

32. C'est l'interprétation des comportements déviants ou plus généralement des nuisances par les promoteurs et leurs vigiles, qui pose problème, variant de l'instauration de l'ordre, la gestion des flux à l'expulsion des personnes marginales.

33. À l'exception de certains profils particuliers comme les mendiants et les ivrognes. En ce sens, nous avons assisté à une situation « gênante » dans le centre commercial Al Manal à Rabat, retranscrite dans notre journal du bord : «C'était une journée de grande affluence à Al Manal. Une foule importante sillonne ses divers couloirs au point qu'il m'était difficile d'y circuler librement. Pendant qu'un agent de sécurité observe son secteur, il reçoit un appel dans son talkie-walkie. “Dans quel secteur elle a été repérée ?” interroge-t-il brutalement. “T'inquiète pas je vais m'en charger tout de suite” répond-il. Il fait signe à son compagnon et disparaissent rapidement dans les couloirs de l'enceinte. Après quelques minutes, ils ramènent avec eux une personne. Visiblement c'était une mendiante. “Je t'avais déjà avertie de ne plus te revoir ici" lui dit le vigile d'un ton nerveux. "Mais fiche-moi la paix, je n'ai fait aucun mal aux gens” répond la mendiante. “C'est toi qui dois me laisser en paix, allez dégage d'ici, la prochaine fois je te mets en tôle” réplique l’agent comme s’il était un vrai policier ! » (Extrait du journal de bord, juin 2010). 
sique), mais cela ne doit pas occulter la présence d'autres formes de « dissuasion » plus subtiles. Celles-ci sont matérialisées, notamment, par les normes contraignantes d'utilisation de ces espaces, les regards des vigiles ou l'accueil inhospitalier des gérants des enseignes de marque à l'égard des personnes « indésirables » rendant ainsi leur expérience du mall très gênante. De telles pratiques concernent aussi bien des individus «non consommateurs » dont la visite se limite à une découverte sans achat, que des « consommateurs » (qui y achètent quelques biens) mais dont le profil et l'apparence physique et vestimentaire ne correspondent pas à l'image recherchée. Il serait alors intéressant, face à ces pratiques dissuasives, d'analyser les formes de résistance visibles et invisibles déployées par ces catégories pour investir ces lieux.

\section{Les résistances pour le mall : les pratiques de contournement pour accéder à un espace " ressource"}

Les résultats issus aussi bien des sondages de fréquentation de la clientèle publiés périodiquement par quelques centres commerciaux de la capitale que de nos propres enquêtes ${ }^{34}$ sur un échantillon de leur public montrent la prédominance nette d'une clientèle « aisée » à fort pouvoir d'achat ${ }^{35}$, dont le profil s'inscrit pleinement dans la cible privilégiée par les promoteurs commerciaux. La proportion dominante de la clientèle « aisée » doit être relativisée et nuancée en fonction des temporalités de fréquentation telles que la fin de la semaine, la fin du mois, les périodes des fêtes et des soldes où la présence d'une foule hétérogène et diversifiée est plus visible. C'est ce que montrent d'ailleurs nos observations de la variation des profils des personnes qui les fréquentent, effectuées sur des durées prolongées. Il est intéressant, à ce sujet, de savoir comment des profils «non ciblés » parviennent à se rendre dans ces lieux sélectifs et excentrés, puis à y pénétrer malgré les barrières physiques et symboliques qui sont dressées à leur encontre. Nos observations montrent que la majorité des visiteurs de ces espaces y accèdent en voiture. Cependant, l'usage d'autres moyens de transport par une petite minorité des visiteurs mérite d'être analysé. En effet, malgré leur faible connexion aux réseaux de transport en commun, ces malls sont également investis par des personnes non motorisées qui déploient pour y accéder tout un ensemble de stratégies. Ainsi, à Marjane Hay Ryad qui est un important centre commercial situé dans une zone excentrée de Hay Ryad, le long d'une rocade et déconnectée du réseau

34. À partir d'un échantillonnage aléatoire effectué dans quatre centres commerciaux sur différents jours de la semaine durant un mois d'enquête, nous avons interrogé une centaine de personnes. Nous avons relevé que plus de $80 \%$ d'entre elles sont des cadres moyens et supérieurs, motorisés et résidant dans les principaux quartiers aisés de l'agglomération (Hay Ryad, Souissi, Agdal, Wifaq, Bettana, etc.).

35. Selon des données fournies par la société gestionnaire de l'enseigne Marjane plus de « $50 \%$ des clients ont des revenus supérieures à $9500 \mathrm{dh}(875 €)$ par mois ». Une moyenne qui varie selon le standing de ces magasins et qui reste très au-dessus de la moyenne nationale (environ 5000 dh soit 465€), voir « La grande distribution au Maroc», fiche de synthèse, missions économiques, Ambassade de France au Maroc, 9 mars 2004. 
de transports en commun, il est intéressant d'observer comment les individus sans voiture s'y rendent en empruntant des bus depuis les quartiers populaires (Takaddoum, Massira 2 à Témara au sud de Rabat, etc.). Bien que la station de bus la plus proche de Marjane Ryad se trouve à plusieurs centaines de mètres impliquant des cheminements piétonniers longs et difficilement praticables, cela n'a pas dissuadé quelques familles non motorisées d'utiliser ce mode d'accès ${ }^{36}$. C'est le cas de la famille Tijari, résidente du quartier de Hay Nahda à Témara, qui se rend occasionnellement à Marjane par bus pour y faire des approvisionnements : «De temps en temps, lors des périodes des soldes, nous venons en famille à Marjane Ryad pour faire les courses dans son immense hypermarché. Nous empruntons le bus qui nous dépose dans une station non loin de Marjane ; c'est notre cousin Aziz, un ouvrier travaillant dans un chantier à Hay Ryad qui nous a montré le chemin à faire pour accéder au centre commercial. Effectivement, c'est un peu dur d'emprunter cette voie surtout durant la saison estivale mais peu importe du moment qu'on passe des moments agréables en famille 37 » fait savoir Rachida, une femme au foyer de 33 ans dont le mari occupe le poste de chauffeur de bus à Témara. L'absence de cheminements piétonniers aménagés reliant Marjane à son environnement proche n'a pas dissuadé non plus les jeunes issus des bidonvilles situés à proximité de l'enceinte commerciale de s'y rendre à pied, ni les ouvriers travaillant dans les chantiers de construction de Hay Ryad. C'est particulièrement le samedi après-midi qu'il est intéressant d'observer l'affluence de ces catégories sociales au sein du supermarché. En plus de ces modes d'accès articulant bus et marche à pied, observés dans les temps de grande affluence, s'ajoutent d'autres moyens d'accès. Si certains jeunes et étudiants utilisent des motocyclettes ou même des bicyclettes pour se rendre dans ces lieux excentrés, d'autres personnes interrogées s'entraident en se cotisant pour louer un grand taxi ou une voiture de transport de marchandises afin de venir s'approvisionner à Marjane Ryad. À ce propos, nous avons pu constater, lors des entretiens, comment certains ménages profitent de la présence d'un membre de la famille ou d'un voisin motorisé qui leur sert de chauffeur jusqu'au centre commercial. Les propos d'Abderrahim, jeune fonctionnaire non motorisé, résidant dans un appartement de logement social à Hay Nahda, à Rabat, le confirment :

« Si je viens ici à Marjane Ryad, c'est grâce à mon voisin motorisé qui m'amène avec lui de temps en temps faire les courses. En fait, nous sommes cinq amis voisins résidant dans le même immeuble. À chaque fin du mois, nous nous cotisons pour lui payer le carburant et nous conduire ensemble à Marjane faire l'approvisionnement mensuel. C'est pour nous une occasion agréable de se retrouver entre amis et de faire l'approvisionnement à moindre coût sans

36. D'autres familles, pour minimiser les frais de transport, utilisent pour le même trajet deux moyens de transport : le bus jusqu'au point d'arrêt le plus proche de Marjane et le petit taxi pour faire le reste du trajet.

37. D’après l'entretien réalisé avec Rachida, femme au foyer, 33 ans. 
prendre chacun un petit taxi qui va coûter plus cher 38 ».

Par ailleurs, si l'épreuve de "déplacement » vers ces malls périphériques est surmontée par un ensemble de pratiques de " contournement », l'accès à leur espace intérieur reste problématique en raison de l'ambiance codifiée et hautement surveillée. Dans cette seconde épreuve "d'accès », les apparences physiques et vestimentaires du public «non ciblé » sont déterminantes pour se faire accepter «sans gêne » dans ces espaces structurés par les images et le paraître.

Comme nous l'avons évoqué plus haut, s’il est exceptionnel, voire rare, d'assister à des situations d'expulsion de personnes appartenant à des catégories sociales jugées "indésirables », il est intéressant de souligner la présence d'autres formes subtiles de dissuasion et "d'intimidation » à leur encontre rendant leur fréquentation parfois gênante ${ }^{39}$. C'est, en effet, dans l'échange des regards qui s'établit entre les vigiles et ces personnes que se cristallisent ces pratiques de dissuasion. Les apparences physiques et vestimentaires servent à ce sujet d'indicateurs privilégiés pour « catégoriser » ces individus et les ranger soit dans le registre du «client potentiel », soit dans celui de l'indésirable.

En fonction de leur paraître et de leurs postures corporelles et gestuelles, ces catégories sociales sont alors l'objet de regards «intimidants » de la part des agents de sécurité, véhiculant l'idée qu'elles ne sont pas les bienvenues dans ces espaces. Ces regards «stigmatisants » peuvent parfois être prolongés en fonction des personnes "suspectes » par des actions d'intimidation comme le fait d'exposer la personne "indésirable » à un test "gênant » de détecteurs de métaux (test qui n'est pas systématiquement généralisé à l'ensemble des clients) ou le fait d'être suivi par un agent de sécurité à l'intérieur de l'espace commercial.

Pour éviter d'être gênés dans leur visite et surmonter l'épreuve du « stigmate », ces « indésirables » (essentiellement des jeunes adolescents et des familles modestes issus des quartiers populaires de l'agglomération), adoptent tout un ensemble de pratiques de détournement. Le soin apporté aux apparences physiques et vestimentaires représente à ce sujet l'une des techniques utilisées pour «fausser » les critères de sélection des vigiles et des gérants des boutiques commerciales. C'est ce que rapporte Saïda, jeune étudiante en économie, issue d'une famille modeste et résidente du quartier populaire Massira 2 à Témara «Il n'est pas question de s'habiller n'importe comment dans ces lieux, sinon tu seras facilement classé dans ces malls habitués à une clientèle aisée 40 ». Cela passe notamment par l'attention portée à la nature des vête-

38. D'après l'entretien réalisé avec Abderrahim, fonctionnaire, 38 ans.

39. Voir à ce sujet Capron G., «L'accès aux espaces publics modernes dans les villes latino-américaines », op. cit.

40. D’après l'entretien réalisé avec Saïda, étudiante, 22 ans. 
ments portés, à la qualité des accessoires utilisés (sac à mains, bijoux, foulard), ainsi qu'à une mise en scène plus décontractée du corps. Le propos de Khadija, jeune célibataire, institutrice dans un collège public à Rabat et résidente dans le quartier populaire de Takkadoum, s'inscrit dans le même sens :

«À chaque fois que je me rends ici au Méga Mall, je porte mes meilleures robes et mes bijoux pour me faire une place dans cet univers de luxe. Autrement, si je m'habille de façon ordinaire, ou pire si je porte des vêtements traditionnels comme la djellaba, je suis sûre que je serai taxée de femme populaire dans ces espaces. [...] Face à une telle situation il y a deux attitudes à faire, soit ne plus revenir ici en disant que c'est fait pour une autre catégorie [l'auto-exclusion], soit revenir avec de nouveaux looks et de nouvelles images de soi [résistance et contournement] ${ }^{41}$ ».

Ces mises en scène du paraître sont particulièrement observées chez les jeunes et surtout les lycéens qui affluent en masse dans ces lieux de consommation. À travers les images qui leur sont souvent renvoyées comme étant des «non-consommateurs » ou des « perturbateurs » en raison de leurs pratiques de déambulation et de drague, ces jeunes multiplient « les ruses » pour véhiculer une autre image, celle d'une jeunesse «branchée » et intéressée par les objets de marque. Cela se manifeste, notamment, par le fait de porter des vêtements de marque ou signés, d'arborer un style et un « look » en phase avec les dernières tendances de la mode, de se faire une coupe de cheveux insolite, ou de manipuler des accessoires de luxe - des téléphones portables dernier cri, des écouteurs, etc.

À l'intérieur de ces univers "branchés ", centrés sur la consommation et où le fait de consommer est synonyme d'appartenance à la clientèle « désirable » du mall, il est intéressant d'observer la présence d'autres pratiques de contournement utilisées par les catégories à faible pouvoir d'achat. Ainsi, en plus de faire des achats dans les périodes des soldes et des promotions pendant lesquelles les articles de marque deviennent plus ou moins accessibles, on peut observer comment certaines groupes sociaux, notamment les jeunes adolescents issus des quartiers populaires, se contentent d'acheter le minimum d'objets, les moins chers possibles, pour avoir l'étiquette de « bon client consommateur »; leurs choix se portent, par exemple, sur un accessoire à un prix abordable dans une boutique de franchise ou sur une glace dans un café haut standing.

Mais, au-delà de ces pratiques de résistance « subtiles » de contournement déployées par ces catégories pour investir ces lieux, il existe d'autres formes de pratiques de résistance de « confrontation » mises en œuvre essentiellement

41. D’après l'entretien réalisé avec Khadija, institutrice, 30 ans. 
par les jeunes adolescents qui s'approprient des lieux interstices (marches, escaliers, couloirs, etc.) et qui font des tournées prolongées dans la galerie marchande malgré le refus des vigiles. Si, au début, les relations avec ces derniers étaient souvent conflictuelles, elles se sont apaisées ${ }^{42}$ au fil du temps au point que ces formes non marchandes d'appropriation de l'espace deviennent tolérées sous certaines conditions telles que réduire le temps d'occupation de ces espaces interstitiels, éviter les endroits de forte affluence, éviter des comportements qui pourraient déranger la clientèle, etc.

Enfin, il est important de souligner que ces pratiques de « résistance » émanant des catégories sociales à faibles revenus sont déployées non pas uniquement contre les normes « contraignantes » du mall, mais aussi contre les regards dissuasifs et «stigmatisants » de sa clientèle aisée. Cela implique de fortes tensions «visuelles » entre ces deux profils qui justifient le recours par ces groupes sociaux, particulièrement les femmes, à d'autres formes de résistance « d'imitation » de la clientèle aisée - le choix de la tenue vestimentaire, l'usage d'une langue étrangère, le port des accessoires de luxe, la mise en scène du corps, etc.

\section{Les résistances par le mall : s'affranchir des normes sociales dans « un espace refuge»}

L'observation récurrente des modalités d'utilisation sociale des centres commerciaux de Rabat-Salé révèle l'existence d'autres formes de résistance lors desquelles ces espaces sont perçus cette fois-ci comme des lieux «parenthèses 43 » ou « refuge » permettant de s'affranchir d'un ensemble de normes sociales et culturelles locales. Il s'agit ici de " résister » non pas contre les formes de dissuasion imposées par le centre commercial, mais contre un ensemble de normes sociales imposées par les milieux d'origine. En raison des images de modernité et de liberté qu'ils mettent en scène, ces malls représentent pour ces groupes sociaux (issus principalement des quartiers populaires) de véritables échappatoires leur permettant de s'affranchir du contrôle social et culturel imposé dans leurs foyers. En effet, leur emplacement excentré, loin de leurs quartiers, leur faible accessibilité sociale, leur anonymat ainsi que leur configuration fermée représentent paradoxalement pour ces groupes sociaux des éléments appréciés pour réaliser « librement » des usages qui sont généralement codifiés dans les lieux de leur provenance. Arborer dans ces espaces de «kharij» un «look » insolite, porter des vêtements jugés souvent choquants dans l'espace public, se faire accompagner par son partenaire de l'autre sexe, fumer une cigarette, etc., représentent quelques manifestations de cette sorte de « libération » provisoire. Certes, ces pratiques juvéniles sont de plus en plus

42. D’après le témoignage apporté par Saïd, un jeune lycéen qui fréquente régulièrement le Méga Mall en compagnie de ses camarades.

43. Gillot G., «Espaces populaires, pratiques intimes : les jardins publics au Caire, à Rabat et à Damas », Géocarrefour, vol. 77, n 3, 2002, pp. 267-274. 
observées dans les espaces publics de l'agglomération, toutefois, dans ces centres commerciaux, elles prennent une acuité toute particulière en raison de leur visibilité et de leur intensité. C'est surtout auprès des femmes et des jeunes filles, dans le rapport qu'elles entretiennent avec ces lieux, qu'il est intéressant d'explorer ces pratiques de résistance. Les images et les valeurs d'émancipation et de liberté que ces équipements affichent, correspondent parfaitement aux attentes et aspirations de cette clientèle ${ }^{44}$. Que ce soit dans les grandes surfaces alimentaires ou dans les espaces de shopping et de loisirs, nous pouvons observer la présence manifeste de groupes de femmes qui se côtoient et déambulent sans gêne dans ces espaces de consommation. L'offre de produits exposés dont la plupart sont des biens importés, la prédominance des personnages occidentalisés dans les spots publicitaires, la prédominance du commerce franchisé (dont la majorité sont des marques étrangères), la commercialisation sans "gêne » de l'alcool, l'affichage parfois des images de femmes « dénudées ", participent à recréer un environnement «importé » qui contraste avec les ambiances et les normes de l'espace extérieur. Cette ambiance occidentalisée leur accorde une plus grande liberté pour s'afficher socialement et se sociabiliser librement. «Quand je fréquente le Méga Mall j’ai toujours cette impression que je suis emportée dans une sorte d’île américaine qui permet de me déconnecter de la réalité locale » affirme Fatiha, jeune étudiante résidente à Hay Nahda, un quartier modeste situé à Témara. Les expressions utilisées de l'île américaine et de la déconnexion sont très parlantes pour signifier l'extraterritorialité du mall par rapport à son entourage.

Les tenues vestimentaires arborées par ces visiteuses, leurs postures plus ou moins décontractées et, parfois, les comportements sociaux de certaines d'entre elles (fumer une cigarette, rester tardivement, s'afficher librement avec son copain) sont quelques-unes des manifestations de cet affranchissement des normes sociales. Ces équipements marchands s'érigent ainsi en véritables espaces « enceintes » ou lieux « limites », selon P.-A. Barthel ${ }^{45}$, qui permettent aux jeunes filles de s'extraire des territorialités du quotidien et de se libérer des codes socialement reconnus dans les espaces publics ou domestiques, voire de les «transgresser ». Comme l'avait bien observé Laure Assaf dans son analyse des pratiques de sociabilité des femmes dans les malls et espaces publics à Abu-Dhabi : «Le caractère global de ces espaces en fait à la fois des lieux respectables, et des lieux où une sociabilité amicale mixte en termes de genre est très légitime 46 ». Le propos suivant de Farida, est éloquent sur la protection symbolique qu'offre à ses yeux le centre commercial :

44. Le Renard A., Femmes et espaces publics en Arabie Saoudite, op. cit.

45. Barthel J-P., "Mondialisation, urbanité et néo-maritimité : la corniche du lac du Tunis », Espace géographique, n², 2006, pp. 129-144.

46. Assaf L., «Espaces vécus et imaginés des rencontres amoureuses aux Émirats arabes unis », op. cit. 
«Avant je ne me sentais jamais à l'aise dans la rue, il me faut toujours être accompagnée par un tiers : soit quelqu'un de la famille, le plus souvent mon petit frère, soit un copain pour qu'on me laisse en paix ; mais ici tout est différent, les gens sont sympas, il y a un respect mutuel surtout pour les femmes, et si quelqu'un ose faire un geste déplacé, il y a toujours des vigiles pour le remettre à l'ordre. À vrai dire je mets rarement les pieds dans les rues pour me promener dans la ville, si ce n'est un passage obligé pour effectuer des achats 47 ».

Ce qu'il faut retenir de son propos, au-delà des expressions significatives associées au mall comme lieu «sélect » et respectueux des femmes, c’est l'image négative associée à la rue comme lieu « mélangé » socialement et non sécurisé qui confirme le processus d'abandon que connaît cet espace au niveau de Rabat-Salé comme lieu public de rencontre et de mixité sociale.

Ces images de protection symbolique associée aux centres commerciaux ont été bien analysées par la sociologue Davis Hannah à propos de la fréquentation des fast-foods " américains » par des femmes marocaines.

«La sécurité que McDonald's offre aux femmes est consciemment et consciencieusement établie et entretenue... La bonne réputation $\mathrm{du}$ lieu attire les filles. Le McDonald's est un lieu public “domestiqué", autorisant des comportements détendus en dehors du foyer. Mais cette sécurité vient aussi de son “américanité”. Les femmes marocaines ont été assez habiles pour s'approprier l'offre de McDonald's et utiliser son américanité pour en faire un lieu public détendu et mixte ${ }^{48} »$.

Ces observations sont confirmées dans d'autres contextes socioculturels et, notamment, dans les travaux conduits par Mona Abaza sur l'accès des femmes dans les centres commerciaux en Égypte ainsi que dans les recherches plus récentes faites par Amélie Le Renard sur les femmes Saoudiennes. L'auteure y souligne que « les malls [à Ryad] sont perçus comme des espaces féminins $[. .$.$] . C'est justement leur accès restreint qui les rend fréquentables$ par les femmes, en plus des règlements limitant cet accès, [leur] agencement pose certaines frontières aux comportements indiscrets ou offensants dans l'enceinte même de cet espace ${ }^{49}$ ».

47. D'après l'entretien réalisé avec Farida, étudiante infirmière, 24 ans.

48. Hannah D-T., "Là où vont les femmes" : notes sur les femmes, les cafés et les Fast Food au Maroc », op. cit.

49. Le Renard A., " Pratiques du shopping mall par les jeunes Saoudiennes. Sociabilité et consumérisme à Riyad ", in Peraldi M, Mermier F. (dir.), Mondes et places du marché en Méditerranée. Formes sociales et spatiales de l'échange, Paris, Karthala, 2010, p. 199. 


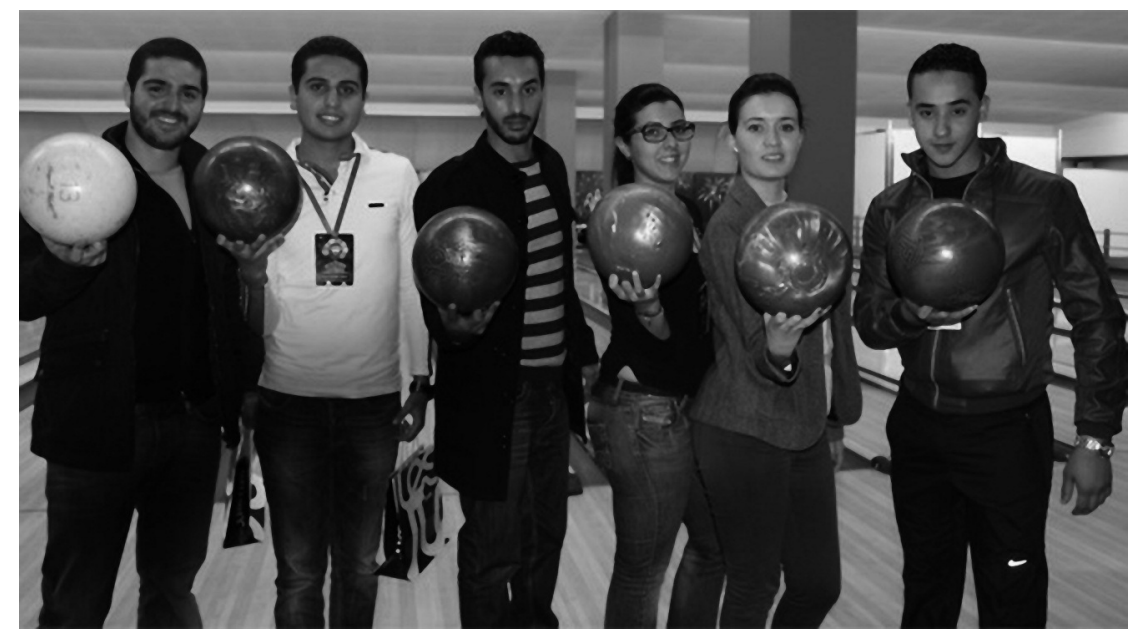

L'espace bowling du Méga Mall investi comme lieu de distraction et de côtoiement mixte pour les jeunes (source : cliché personnel 2009)

En plus de ces sociabilités féminines, des coprésences mixtes réunissant des jeunes des deux sexes sont également récurrentes dans ces malls. Le cadre fermé de ces espaces et les images d'ouverture et de modernité qu'ils incarnent auprès des jeunes Rbatis, favorisent de tels côtoiements. C'est ce que pointe Leila, étudiante originaire du quartier Tabriquet à Salé : « les gens admettent plus facilement qu'on se voie (elle et son ami) dans le centre commercial que de se rencontrer dans la rue 50 ».

En effet, il existe dans ces lieux des coins discrets et intimes qui sont réservés et aménagés pour favoriser de telles rencontres (bowlings, cafés branchés, fast-foods, etc.). Leur ambiance tamisée et les prix qui y sont pratiqués participent à favoriser ces formes de côtoiement. Enfin, un autre élément par lequel peut être appréciée cette résistance à l'encontre des normes sociales et culturelles locales se rapporte à la consommation des boissons alcoolisées, notamment dans les centres commerciaux alimentaires. Dans les rayons d'alcool à Marjane Ryad par exemple, il est impressionnant, sinon frappant, d'observer comment des jeunes et des femmes célibataires achètent de l'alcool ${ }^{51}$ et ce, au vu et au su d'autres nombreux clients. Si ces pratiques de consommation se font en catimini ailleurs - dans les bars, les clubs privés, ou les dépôts de vente d'alcool de proximité aux façades souvent aveugles -, ici elles sont complètement visibles et prennent un caractère tout à faire ordinaire. Les grandes surfaces de ces centres commerciaux sont en effet associées à l'acquisition des boissons alcoolisées. Elles sont préférées par rapport aux points de vente de

50. D’après l'entretien réalisé avec Leila, étudiante, 20 ans.

51. À partir de 2011, les gestionnaires des hypermarchés Marjane ont pris la décision d'arrêter la commercialisation de l'alcool dans les rayons pour des considérations sécuritaires. 
quartier. Leur éloignement par rapport aux quartiers de résidence des clients évite ainsi aux consommateurs de ces boissons d'être identifiés par les voisins ; de plus, l'ambiance occidentalisée de ces espaces, compatible avec la consommation de ces boissons, explique en partie cette préférence. C’est ce que rapporte d'ailleurs Mourad, jeune architecte travaillant au sein d'une administration publique et habitant le quartier Océan, non loin du centre-ville de Rabat : «Lorsque je veux acheter de l'alcool, c'est essentiellement dans ces grandes surfaces que je m'approvisionne. C’est plus organisé, plus pratique et surtout plus toléré par les gens. C'est fini ces achats clandestins qu'on faisait auparavant dans les magasins de vin de proximité 52 ».

Ce qui attire notre attention sur ces différentes images et significations associées au mall par les différentes personnes interrogées, c'est le caractère paradoxal du mall comme étant à la fois un lieu de contrôle régi par un dispositif de surveillance et de régulation des pratiques sociales et un lieu de liberté et de transgression des normes locales. On se demande alors si ce n'est pas sa configuration hautement surveillée et sécurisée - et non principalement son offre marchande et son ambiance ludique - qui est la plus appréciée par les jeunes Rbati(e)s.

\section{Les résistances contre le mall: les pratiques de contestation contre un espace « intrus »}

Une dernière forme de résistance, observée dans les centres commerciaux de Rabat-Salé, se rapporte aux mobilisations et aux mouvements de contestation dont ils sont la cible dans certains quartiers populaires de l'agglomération. Elle renvoie plus exactement à la dimension idéologique et culturelle des appréciations qui émanent de certaines personnes sur ces structures marchandes associées à « l'Occident».

Si certaines d'entre elles ont exprimé des appréciations positives, inscrites dans le registre de la modernité incarnée par ces espaces, d'autres pointent, au contraire, leur caractère exogène en relation avec la culture locale justifiant les attitudes de condamnation qui leur sont adressées.

Perçus souvent comme des espaces « occidentalisés », véhiculant un ensemble de symboles en rupture avec la culture et l'identité locales, ces malls suscitent des réactions négatives qui peuvent évoluer parfois vers des mouvements de contestation de la part d'associations culturelles locales. Ces réactions se traduisent par plusieurs types d'attitudes ${ }^{53}$ qui forment un continuum allant des résistances « muettes » comme les pratiques d'auto-exclusion et de

52. D’après l'entretien réalisé avec Mourad, architecte, 32 ans.

53. Fournier S., «Consumer resistance: societal motivations, consumer manifestations, and implications in the marketing domain ", Advances in Consumer Research, vol. 25, 1998, pp. 88-90. 
non-fréquentation du centre commercial jusqu'aux comportements de réduction d'achat (stratégies d'adaptation, "downshifting ») pour aboutir aux résistances de contestation telles que les plaintes et les sit-in. Les auteurs de ces attitudes mettent en avant une série de motifs et d'images associés aux centres commerciaux renvoyant tantôt à leurs effets d'aliénation et d'acculturation, tantôt à l'hyperconsommation et à l'endettement des ménages qu'ils impliquent. Ce sont les symboles politiques et identitaires associés à ces espaces qui reviennent fréquemment dans les propos soulevés par la plupart des personnes interrogées.

Si certains mobilisent dans ce registre politique les symboles de l'impérialisme et de la domination économique incarnés par ces espaces qui abritent des franchises appartenant à des firmes internationales américaines (McDonald's, Pizza Hut, Nike) ${ }^{54}$, d'autres mobilisent les symboles religieux pour contester la manière par laquelle ils portent atteinte aux valeurs morales locales.

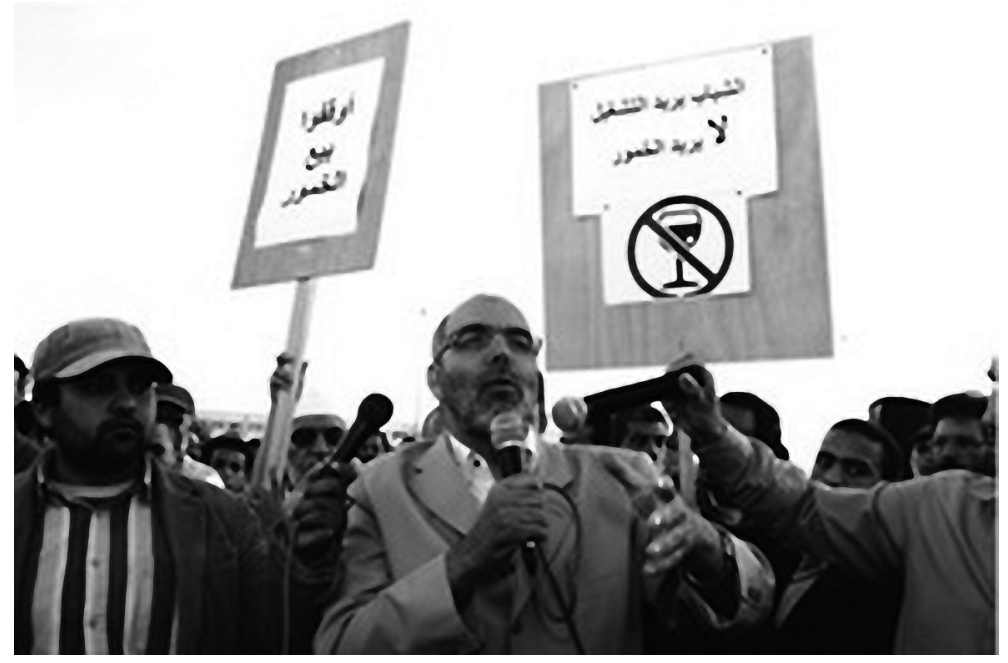

Exemple de mobilisations sociales devant l'entrée principale du centre commercial du Salé (source : Journal Attajdid, 2010)

La consommation de l'alcool et de la viande porcine prohibée pour les Marocains musulmans, aussi bien par la loi ${ }^{55}$ que par la religion, l'affichage des images jugées « choquantes » du corps féminin et l'incitation au côtoiement des hommes et des femmes, sont autant d'éléments qui suscitent pour

54. Justifiant les projets d'attentats dont quelques centres commerciaux du Grand Rabat et leurs enseignes internationales ont été les cibles. On peut mentionner ici le projet d'attentat envisagé par deux sœurs à l'encontre d'un centre commercial à Rabat. Le choix de cet espace comme «cible » a été justifié par le fait qu'il était considéré comme un lieu de débauche mais aussi comme symbole de l'hégémonie occidentale (" Procès des jumelles terroristes-apprenties ", Aujourd'hui, 30 septembre 2003).

55. Datant de 1967, une loi interdit à tout exploitant de vendre et d'offrir des boissons alcoolisées à des Marocains musulmans. 
certains des sentiments de rejet et d'indignation. S'agissant de la commercialisation de l'alcool dans les grandes surfaces périphériques de l'agglomération Rabat-Salé, il faut souligner la multiplication des sit-in et des mouvements de contestation initiés par des associations culturelles religieuses 56 à Rabat et à Salé qui se sont mobilisées contre la vente de ces boissons sans aucune restriction. Initiées le plus souvent par des associations de quartiers populaires de Salé, ces protestations se sont effectuées devant les principaux accès de Marjane et Carrefour de Salé plaidant pour l'interdiction immédiate de la vente d'alcool et menaçant même du boycott de fréquentation de ces structures. « Les grandes surfaces dérangent le PJD » tel est le titre affiché par un journal marocain pour souligner les propos de condamnation et les mobilisations multipliées par ce parti (Parti de la justice et du développement) et ses députés contre la mise en vente d'alcool dans les grandes surfaces. C'est particulièrement à Salé où se concentre une bonne partie de ces associations locales que les réactions contre le nouveau centre commercial périphérique de l'enseigne Carrefour installé non loin du quartier populaire de Sidi Moussa ont été médiatisées. Des slogans ont été affichés et proclamés dans ces mouvements de protestation "Carrefour, arrêtez l'alcool », « la jeunesse cherche de l'emploi et non du vin » pour exprimer des contestations à la fois d'ordre culturel (vente de l'alcool), économique (emploi des jeunes) et social (pallier la délinquance) contre l'arrivée de cette structure commerciale. C’est ce que nous rapporte Amine, un jeune membre associatif, habitant du quartier populaire de Sidi Moussa à Salé et ayant participé au sit-in contre l'enseigne de Carrefour : "Ce sont les pauvres habitants et les jeunes de ce quartier populaire de Sidi Moussa qui sont les victimes de l'arrivée de ces espaces commerciaux. On n'a rien gagné de ces structures présentées comme opportunités d'investissement. Ils se font des fortunes énormes à partir des bourses modestes de la ville, ils contribuent au surendettement des ménages pauvres et le pire est qu'ils participent à impulser les taux de délinquance et de marginalité chez les jeunes par la commercialisation en plein public de l'alcool ${ }^{57}$ ». Sur ce même sujet de la vente de l'alcool dans ces surfaces modernes, il faut surtout mentionner la controverse qu'a suscitée « la fatwa 58 » d'un imam marocain, président du mouvement islamiste «Attawhid wal Islab» (Ahmed Raissouni), connu au Maroc et dans le monde arabe, qui a prohibé l'achat et l'approvisionnement de l'alcool dans les grandes surfaces. Lancée à partir du mois du juin 2010, cette fatwa a suscité de vives réactions de la part de certaines associations qui se sont soulevées contre l'idée de dissuader les gens à fréquenter ces structures commerciales bien qu'ils n'aient pas l'intention d'acheter du vin. À ce titre également, les propriétaires de la chaîne Marjane se

56. Soutenues le plus souvent par des partis et des mouvements islamistes.

57. D'après l'entretien réalisé avec Amine, membre associatif, 39 ans.

58. Il s'agit de l'avis personnel d'un imam sur une question touchant la vie quotidienne des musulmans et qui n'a pas été abordée par les textes religieux (Coran et hadith). La fatwa de Raissouni a été présentée dans un quotidien local et largement diffusée dans les réseaux sociaux. 
sont mobilisés contre ces propos pour rappeler que la vente de l'alcool est réservée aux étrangers en total respect de la loi en vigueur ${ }^{59}$. En dépit de ces propos « rassurants ", il faut reconnaître les effets qu'a entraînés cette fatwa sur la fréquentation des centres commerciaux à vocation alimentaire tels que les magasins de Marjane, Acima et Label'Vie de Rabat. Certains clients du Marjane préfèrent se rendre désormais à Aswak Assalam dont le concept commercial repose, entre autres, sur l'interdiction de la commercialisation de l'alcool et de la viande porcine en respect de la culture religieuse locale. Le concept de marocanité, tel qu'il a été privilégié par les promoteurs de ce centre commercial, a eu alors un écho positif auprès de nombreuses familles qui préfèrent désormais s'approvisionner dans un cadre qui répond parfaitement aux spécificités culturelles locales : " Je viens ici à Marjane de temps en temps avec mon fils aîné et ses enfants, je trouve honteux, voire scandaleux, de voir comment des espaces situés dans ce pays vendent de l'alcool aux jeunes sans aucun complexe et tolèrent des pratiques qui portent atteinte à leurs valeurs morales. Je vois ces choses et je me pose la question si l'on est vraiment dans un pays où l'islam représente, d'après la constitution, la religion officielle. Depuis quelque temps, mes approvisionnements je les fais en famille à Aswak Assalam, là au moins il y a un respect pour nos valeurs et pour les familles marocaines 60 » affirme Mohammed, retraité et résidant dans l'ancien quartier de Diour Jemaa à Rabat. L'attitude de condamnation et de boycott de Mohammed et d'autres personnes contre le centre commercial de Marjane représente, contrairement aux mouvements de protestation initiés par les collectifs d'associations, des réactions individuelles et isolées qui s'inscrivent dans le registre de la résistance muette.

À ces réactions muettes, s'ajoutent d'autres formes de résistances mises en œuvre par des personnes qui utilisent les divers moyens de communication pour exprimer leur condamnation et leur indignation contre ces malls. C'est le cas d'une personne anonyme qui a communiqué dans un journal quotidien son indignation contre la vente de l'alcool dans les grandes surfaces de Marjane : "Je vous écris en tant que citoyen marocain, pour exprimer mon indignation quand j'ai trouvé dans ma boîte aux lettres le dernier dépliant des magasins Marjane... Grande fut mon indignation et mon amertume devant l'effronterie et l'audace irrévérencieuse de ces magasins en trouvant la page des Vins et liqueurs, nous invitant à profiter des petits prix proposés, manquant de peu nous conseiller avec quel vin ou quelle liqueur accompagner le Kabab de l'Aïd... L'alcool est vendu librement dans ces surfaces, j'en conviens. Chacun est libre de boire ou pas, mais dans le respect du sentiment de l'autre. Alors de grâce, Messieurs de Marjane, arrêtez de nous christianiser. Ceci n'est

59. Un avis est en effet affiché dans l'entrée de la grande surface Marjane précisant que la consommation d'alcool est interdite par la loi aux Marocains. Mais il est curieux de constater que la file d'attente devant la caisse du rayon de l'alcool est composée majoritairement de consommateurs marocains. L'avis affiché n'est en réalité qu'une formalité administrative.

60. D’après l'entretien réalisé avec Mohammed, retraité, 66 ans. 
pas la réaction d'un intégriste mais d'un citoyen musulman 61 ». Mais au-delà de ces communiqués publiés dans les journaux et médias locaux, il est impressionnant d'observer aujourd'hui le foisonnement des réactions faites par les internautes dans les réseaux sociaux, les forums et blogs personnels sur ces structures commerciales. Si certains mettent en avant les symboles de modernité et de liberté qu'ils incarnent pour la société locale, d'autres n’hésitent pas à pointer son effet destructeur et nuisible sur l'identité locale et le pouvoir d'achat des ménages marocains. En somme, l'analyse de ces différentes images révèle comment le centre commercial suscite des significations contrastées, révélant ainsi l'hétérogénéité des valeurs et des références idéologiques de la jeunesse marocaine.

\section{Les centres commerciaux de l'agglomération Rabat-Salé : de « non-lieux » sélectifs à des lieux « ressources » pour les jeunes Rbati(e)s}

À l'instar de ce qu'avait révélé Clifford Geertz ${ }^{62}$ dans son analyse très fine du Souk de Séfrou au Maroc, notre analyse des centres commerciaux de Rabat-Salé et de leur réception sociale par les Rbatis a montré comment ces équipements représentent des « espaces miroirs » révélateurs des tensions et des paradoxes sociaux et culturels qui traversent la capitale administrative. Ces vitrines de la mondialisation sont, en effet, l'expression de la montée de la classe moyenne au Maroc, mais aussi de l'agrandissement considérable de l'écart séparant les classes aisées des catégories sociales modestes. À travers les images et les symboles de richesse et d'opulence qu'ils mettent en scène, ils suscitent des représentations contrastées et des formes de résistance hétérogènes entre ceux qui en font des lieux de désintégration suscitant l'indignation et la condamnation (résistance de contestation) et ceux qui les perçoivent au contraire comme de nouveaux lieux d'intégration et d'appartenance urbaine suscitant des appropriations (résistance d'intégration).

Les images associées à ces nouvelles structures marchandes oscillent de manière paradoxale entre lieux de contrôle régis par des dispositifs de surveillance et de régulation des pratiques sociales et lieux de liberté et de transgression des normes locales. On se demande alors si ce n'est pas cette image « paradoxale » qui explique l'attractivité de ces malls auprès des jeunes Rbati(e)s. L'étude des modes d'utilisation de ces espaces marchands par ces catégories juvéniles permet par ailleurs de se distancier et de nuancer un certain nombre de propos qui les assimilent souvent à des «non-lieux » ou à des enclaves urbaines qui seraient totalement déconnectées de leur entourage et favoriseraient la fragmentation spatiale et sociale. Cherchant dans la même

61. Un citoyen marocain scandalisé par les dépliants des grandes surfaces, Maroc Hebdo, http://www.maroc-hebdo.press.ma/Site-Maroc hebdo/archive/Archives, février 2006, consulté le 11 juin 2011.

62. Geertz C., Le Souk de Sefrou, sur l'économie de bazar (1979), trad. par Céfaï D., Paris, Éditions Bouchène, 2003. 
optique à remettre en question l'attribut de non-lieu par lequel sont désignés ces espaces, Bertrand Lévy souligne que « sur le plan de la mémoire et du souvenir, ces non-lieux [à savoir, les gares, les aéroports, les centres commerciaux, les fast-foods] pourront se doubler d'une dimension affective et être ressentis comme des lieux. Du moment où un non-lieu acquiert une mémoire liée au vécu, il accumule une charge existentielle, identitaire et symbolique, qui est susceptible de le faire passer au stade de lieu 63 ». La manière avec laquelle ces malls sont investis et appropriés par les jeunes Rbatis issus des quartiers populaires de l'agglomération qui parviennent à y accéder et à y effectuer des pratiques non marchandes de déambulation, révèle tout un ensemble de formes de résistances et d'arts de faire à travers lesquels ces jeunes parviennent à transformer ces équipements conçus initialement comme des « non-lieux » en de véritables lieux-ressources qui leur sont profitables. Il est ainsi intéressant d'observer à ce propos comment la dimension marchande de rentabilité prônée et véhiculée initialement par la plupart des promoteurs de ces malls semble loin d'être réalisée. Elle est de plus en plus nuancée au regard de la rude concurrence qui caractérise le secteur de la grande distribution au Maroc, mais aussi de l'ampleur des pratiques déambulatoires et non marchandes croissantes observées dans ces lieux. En ce sens, on peut noter la décision récente du promoteur du centre commercial Marjane Ryad d'aménager une aire de stationnement réservée aux petits taxis et de prévoir des cheminements piétonniers dans l'entourage de l'enceinte commerciale. Le même propos peut concerner les pratiques de déambulation qui étaient considérées comme des comportements non marchands indésirables. Ces pratiques sont de plus en plus reconnues par les gestionnaires de ces centres commerciaux. «Bon nombre de visiteurs viennent ici pour flâner et explorer notre mall. Ils font le tour de la galerie commerciale. Bien entendu, on tolère ce genre de pratiques car elles nous rappellent les usages qui ont fait l'originalité des espaces commerciaux de la ville. Et puis les gens sont libres de faire ce qu'ils veulent du moment que cela ne gêne pas le fonctionnement de notre magasin 64 ».

En définitive, ce propos interpelle sur le rôle que peuvent assumer désormais ces structures commerciales dans la ville, qui se présentent d'ailleurs parmi les rares lieux de rassemblement et de côtoiement à l'échelle de la capitale, dans l'avènement et la construction de nouvelles formes d'être ensemble 65 à Rabat.

63. Lévy B., "La place urbaine en Europe comme lieu idéal », in Ghervas S., Rosset F. (dir.), Lieux d'Europe, Paris, Maison des sciences de l'homme, 2008, p. 70.

64. D’après l'entretien réalisé avec le directeur du Méga Mall en 2010. Le même constat a été évoqué par le directeur d'Aswak Assalam qui souligne que " la flânerie est un phénomène de plus en plus récurrent dans le magasin. Les parents viennent ici souvent en compagnie de leurs enfants. En réalité ce phénomène ne gêne pas du tout le fonctionnement du magasin puisque notre concept même est basé sur la notion de l'hyper famille et la distraction familiale » (L. Dakch, directeur du Magasin Aswak Ryad, 2009).

65. Navez-Bouchanine F. (dir.), La fragmentation en question: des villes entre fragmentation spatiale et fragmentation sociale?, Paris, L'Harmattan, 2002. 